\title{
Neutralizing and protective epitopes of the 2009 pandemic influenza H1N1 hemagglutinin
}

\author{
Falko Schmeisser, Rachel Friedman, Joseph Besho, Vladimir Lugovtsev, Jackeline Soto, Wei Wang, \\ Carol Weiss, Ollie Williams, Hang Xie, Zhiping Ye, Jerry P. Weir
}

Division of Viral Products, Center for Biologics Evaluations and Research, Food and Drug Administration, Bethesda, MD, USA.

Correspondence: Jerry P. Weir, Division of Viral Products, Center for Biologics Evaluations and Research, Food and Drug Administration, 8800

Rockville Pike, Bethesda, MD 20892, USA. E-mail: jerry.weir@fda.hhs.gov

Accepted 8 September 2012. Published Online 5 November 2012.

\begin{abstract}
Aims and Methods To facilitate antigenic characterization of the influenza A 2009 pandemic H1N1 [A(H1N1)pdm09]

hemagglutinin (HA), we generated a panel of murine monoclonal antibodies (mAbs) using as the immunogen mammalian-derived virus-like particles containing the $\mathrm{HA}$ of the

A/California/04/2009 virus. The antibodies were specific for the A/California/04/2009 HA, and individual mAbs suitable for use in several practical applications including ELISA,

immunofluorescence, and Western blot analysis were identified.

Results and Conclusions As the panel of mAbs included antibodies with hemagglutination inhibition (HI) and virus neutralizing activities, this allowed identification and characterization of potentially important antigenic and neutralizing epitopes of the A/California/04/2009 HA and comparison of those epitopes with the HAs of other influenza viruses including seasonal H1N1 viruses as well as the A/South
\end{abstract}

Carolina/1918 and A/New Jersey/1976 H1N1 viruses. Three $\mathrm{mAbs}$ with the highest $\mathrm{HI}$ and neutralizing titers were able to provide passive protection against virus challenge. Two other mAbs without $\mathrm{HI}$ or neutralizing activities were able to provide partial protection against challenge. HA epitopes recognized by the strongest neutralizing mAbs in the panel were identified by isolation and selection of virus escape mutants in the presence of individual mAbs. Cloned viruses resistant to HI and antibody neutralization were sequenced to identify mutations, and two unique mutations (D127E and G155E) were identified, both near the antigenic site Sa. Using human post-vaccination sera, however, there were no differences in $\mathrm{HI}$ titer between

A/California/04/2009 and either escape mutant, suggesting that these single mutations were not sufficient to abrogate a protective antibody response to the vaccine.

Keywords Hemagglutinin, monoclonal antibody, protective epitopes.

Please cite this paper as: Schmeisser et al. (2013) Neutralizing and protective epitopes of the 2009 pandemic influenza H1N1 hemagglutinin. Influenza and Other Respiratory Viruses 7(3), 480-490.

\section{Introduction}

The novel swine-origin influenza H1N1 virus that emerged in 2009 as the first influenza pandemic of the 21st century differed in several notable ways from influenza H1N1 viruses that were circulating in the human population at that time. ${ }^{1}$ This virus was similar in genetic make-up to triple-reassortant swine influenza viruses that had circulated in swine during the preceding 10 years but that only had sporadically infected humans. ${ }^{2}$ The $H A, N A, N P, M$, and NS gene segments of the triple-reassortant swine influenza viruses originated from classical swine lineage viruses; the $P A$ and $P B 2$ gene segments were of North American avian origin; the $P B 1$ gene segment had a human influenza virus origin. However, in the triple-reassortant pandemic 2009 H1N1 [A(H1N1)pdm09] virus, the NA and $M$ genes had a Eurasian swine origin that had not been previously detected in swine influenza viruses isolated in the United States. ${ }^{3,4}$
Because of the importance of antibodies to the virus hemagglutinin (HA) for protection against influenza, the antigenic relationship and relatedness of the $\mathrm{A}(\mathrm{H} 1 \mathrm{~N} 1) \mathrm{pdm} 09$ virus HA to the HA of seasonal influenza viruses circulating in 2009 was of particular significance. H1N1 viruses circulated and drifted antigenically in humans from the 1918 pandemic until replaced by H2N2 in the 1957 pandemic. Upon its reemergence in 1977 and until 2009, further antigenic drift of the H1N1 HA occurred. Although having a common origin with the $1918 \mathrm{H} 1 \mathrm{~N} 1$, the swine H1N1 HA remained relatively stable antigenically from 1930 until the late 1990s, when the swine triple-reassortants likely emerged. Subsequently, there was an increased genetic and antigenic diversity in the swine H1N1 HA. ${ }^{5}$ The net result of the two different evolutionary paths was a substantial antigenic divergence between circulating strains of seasonal H1N1 in humans and the swine H1N1 viruses that gave rise to the 2009 H1N1 pandemic virus. 
Several reported studies have attempted to assess the extent of cross-reactivity between antibodies to the 2009 H1N1 pandemic virus and recent seasonal influenza viruses, and to earlier H1N1 isolates such as those from the 1918 H1N1 pandemic or the 1976 swine influenza outbreak in New Jersey. In most cases, there appears to be some level of $2009 \mathrm{H} 1 \mathrm{~N} 1$ pandemic cross-reactive antibody in persons older than 55-60 years of age, corresponding with exposure to H1N1 viruses before the 1957 pandemic. $^{6-8}$ Further, cross-reacting antibodies to the $\mathrm{A}(\mathrm{H} 1 \mathrm{~N} 1) \mathrm{pdm} 09$ virus appear associated with 1976 swine influenza vaccination, ${ }^{8-10}$ consistent with the relatively close genetic relationship between those viruses. On the other hand, there are some conflicting data regarding the cross-reactivity of the 2009 pandemic virus and recent seasonal H1N1 viruses. Some studies have reported little, if any, cross-reactivity, ${ }^{6,11,12}$ but others have indicated some limited antibody cross-reactivity. ${ }^{13-17}$ Differences in study design and methodologies likely account for such different conclusions, but overall it appears that cross-reactivity of antibodies to the $\mathrm{A}(\mathrm{H} 1 \mathrm{~N} 1)$ pdm09 $\mathrm{HA}$ and seasonal H1N1 HA is at least limited.

To facilitate antigenic characterization of the $\mathrm{A}(\mathrm{H} 1 \mathrm{N1})$ pdm09 HA, we generated a panel of murine monoclonal antibodies (mAbs) to the HA of A/California/04/2009. These hybridoma clones were generated using mammalian-derived influenza virus-like particles (VLP) containing the $\mathrm{HA}$ of the $\mathrm{A} / \mathrm{California} / 04 / 2009$ virus as the immunogen. We describe the initial characterization of these mAbs, the identification of several antibodies with neutralizing and hemagglutination activity, and the evaluation of antibodies for their protective effect in mice when administered passively before influenza virus challenge. The HA epitopes recognized by strongly neutralizing mAbs were identified by the isolation of virus escape mutants. This panel of mAbs allowed comparison of antigenic and neutralizing epitopes between the A/California/04/2009 HA and the HAs of related H1N1 viruses and may help identify important HA epitopes involved in future antigenic drift.

\section{Materials and methods}

\section{Cells and viruses}

Influenza viruses were propagated in 9-day-old specific pathogen-free embryonated chicken eggs. Modified vaccinia virus Ankara (MVA) vectors expressing influenza HA have been described previously ${ }^{18,19}$ and were propagated in chicken embryo fibroblasts (CEF) cells and titered by plaque assay on DF-1 cells. All methods for the generation, propagation, and preparation of recombinant viruses were essentially as described by Earl et al. ${ }^{20}$ Influenza VLP containing only the HA from the $\mathrm{A}(\mathrm{H} 1 \mathrm{~N} 1) \mathrm{pdm} 09$ virus were prepared by MVA vector infection of Vero cells and puri- fied on $10-45 \%$ sucrose gradients as previously described. ${ }^{18}$ Primary CEF cells were obtained from Charles River Laboratories, Wilmington, MA, USA; DF-1 and Vero cells were originally obtained from ATCC (CRL-12203 and CCL-81, respectively). All cells were maintained in Dulbecco's modified Eagle medium supplemented with $10 \%$ FBS (HyClone, Logan, UT, USA), 2 mm l-glutamine, and $50 \mu \mathrm{g} / \mathrm{ml}$ gentamicin.

\section{mAbs to A/California/4/2009 HA}

Monoclonal antibodies to A/California/4/2009 HA were prepared by Precision Antibody (Columbia, MD, USA). $\mathrm{BALB} / \mathrm{c}$ mice were immunized and boosted with mammalian-derived VLP containing only the influenza A/California/4/2009 HA. When antibody titers were $>1: 50000$ in an HA VLP ELISA analysis, spleen cells were fused with a myeloma line. From more than 35 initial hybridoma clones, 11 were selected and expanded for further testing. $\mathrm{mAbs}$ from tissue culture supernatant or from mouse ascites fluids (Harlan Bioproducts for Science, Inc., Madison, WI, USA) were purified by Protein G chromatography.

\section{Passive transfer of $\mathrm{mAbs}$ and animal challenge}

Specific pathogen-free female BALB/c mice (Taconic Farms, Germantown, NY, USA) were housed in a BSL2 facility at CBER/FDA with ad libitum access to water and feed. All antibody transfers, and challenges were performed in accordance with an approved animal protocol; the procedure was similar to that described previously. ${ }^{9}$ At the age of 9-10 weeks, mice in groups of five were injected intravenously (i.v.) with $100 \mu$ l per mouse of either PBS or mAbs $(0.5 \mathrm{mg} / \mathrm{ml})$. At 4 hours after antibody transfer, mice were challenged intranasally with $1 \mathrm{LD}_{50}$ of wild-type A/Mexico/4108/2009 virus (50 $\mu \mathrm{l} /$ mouse) under light anesthesia of isofluorane. Body weight and mortality were monitored daily for 2 weeks after infection.

\section{Immunofluorescence analysis}

MDCK cells were infected with A/California/04/2009 over a range of multiplicities in 12-well plates. At 3 days postinfection, cells were fixed with $4 \%$ paraformaldehyde (1 hour, room temperature) in Tris-buffered saline (TBS). After washing with TBS, cells were permeabilized with $0 \cdot 1 \%$ Triton-X-100 in TBS for 15 minutes, followed by paraformaldehyde quenching with TBS supplemented with $100 \mathrm{~mm}$ glycine. After washing, cells were incubated with primary antibody $(2 \mu \mathrm{g} / \mathrm{ml}$ in $\mathrm{TBS} / 20 \%$ fetal bovine serum $/ 0 \cdot 1 \%$ Triton-X-100) for 1 hour. Goat anti-mouse FITC-conjugated secondary antibody (Southern Biotech, Birmingham, AL, USA) was used for detection. Control antibodies were a mouse monoclonal antibody against influenza NP (BEI Resources, Manassas, VA, USA) and a monoclonal antibody to $\beta$-galactosidase (Promega, Madison, 
WI, USA). Immunostained cells were observed using a Zeiss Axiovert $40 \mathrm{CFL}$ microscope with an X-Cite Series 120 Q fluorescent light source. Images were acquired using Carl Zeiss Imaging System software and an AxioCam MRm camera and exported in TIFF format.

\section{Western blot analysis}

Inactivated influenza viruses were analyzed by sodium dodecyl sulfate (SDS)-polyacrylamide gel electrophoresis and western blot analysis as previously described. ${ }^{18}$ Detection was by chemiluminescence (SuperSignal West Dura extended-duration substrate; Pierce, Rockford, IL, USA) using a LAS-3000 imager (Fujifilm Medical Systems, Stamford, CT, USA).

\section{ELISA}

Sucrose gradient purified VLPs or inactivated influenza viruses were used as capture antigens in ELISA as previously described. ${ }^{18}$ The endpoint titer was defined as the highest dilution of antibody that gave an absorbance $(405 \mathrm{~nm})$ value that was greater than that of a matched dilution of control antibody of the same isotype and was also $>0 \cdot 050$.

\section{Hemagglutination inhibition assay}

The hemagglutination inhibition assay (HI) was performed in 96-well plates (U-bottom) by a standard method, essentially as described previously ${ }^{21}$ using $0.5 \%$ chicken red blood cells suspended in PBS ( $\mathrm{pH} \mathrm{7 \cdot 2).}$

\section{Human serum samples}

Paired serum samples (pre- and post-vaccination) from healthy human volunteers vaccinated with 2010-2011 Southern Hemisphere trivalent influenza vaccine were kindly provided by Dr. Masato Tashiro (National Institute of Infectious Diseases, Tokyo, Japan) in support of the WHO consultation on annual influenza vaccine strain selection. A written informed consent was provided to all volunteers at the beginning of the enrollment by each physician according to the guidelines of the corresponding institutional review board. Healthy adults (20-58 years old) and elderly (62-100 years old) subjects were given a single dose (15 $\mu \mathrm{g} \mathrm{HA} /$ strain/dose) of 2010-2011 trivalent inactivated vaccine intramuscularly. The vaccine was formulated according to the Southern Hemisphere seasonal vaccine composition containing A/California/07/2009 (H1N1), A/Victoria/210/2009 (H3N2), and B/Brisbane/60/2008) as recommended by WHO. Sera were collected at the time of enrollment and 21 days after vaccination.

\section{Virus neutralization assay}

The influenza virus neutralization assay (VN) was performed essentially as described previously. ${ }^{22}$ The presence of virus was detected using biotin-conjugated antibody to influenza NP (clone A; Milipore, Billerica, MA, USA) followed by HRP-labeled Streptavidin (KPL).

\section{HA-pseudotype neutralization assay}

Construction of retroviral pseudotypes expressing the influenza HA of A/California/04/2009, A/New Caledonia/20/1999, A/Solomon Islands/3/2006, A/Brisbane/59/ 2007, and A/New Jersey/1976 has been described previously. ${ }^{10,23}$ The A/South Carolina/1918 HA expression plasmid was obtained from Dr. Gary J. Nabel (National Institutes of Health, Bethesda, MD, USA). Briefly, HA pseudotypes were produced in $293 \mathrm{~T}$ cells by cotransfection of the retroviral packing vector (pCMVDR8.2), luciferase reporter vector ( $\mathrm{pHR}$ 'CMV-Luc), human airway trypsinlike protease expression plasmid ( $\mathrm{pHAT}$ ), and HA expression plasmid, as described. ${ }^{24}$ At 18 hours post-transfection, cells were fed fresh medium containing $7 \mathrm{mU} / \mathrm{ml}$ of bacterial neuraminidase from Vibrio cholera (Sigma, St. Louis, $\mathrm{MO}$, USA) to induce the release of HA pseudotypes from the producer cells. HA pseudotypes were collected $48 \mathrm{~h}$ post-transfection, filtered through a $0 \cdot 45-\mu \mathrm{m}$ low protein binding filter, and used immediately or stored at $-80^{\circ} \mathrm{C}$. HA-pseudotype titers were determined by measuring luciferase activity in infected cells and were expressed as relative luminescence units per milliliter of HA-pseudotype supernatants $(\mathrm{RLU} / \mathrm{ml})$. For neutralization assays, HA pseudotypes containing $\sim 15 \mathrm{ng} / \mathrm{ml}$ of $\mathrm{p} 24$ antigen were incubated with antibody samples for 1 hour at $37^{\circ} \mathrm{C}$, and then $100 \mu \mathrm{l}$ of pseudovirus and antibody mixture was inoculated onto 96-well plates that were seeded with $2 \times 10^{4} 293 \mathrm{~T}$ cells/well 1 day prior to infection. The antibody dilution causing a 95\% reduction in RLU compared with control (IC95-neutralizing antibody titer) was used as the neutralization endpoint titer. ${ }^{25}$ IC95 was calculated using GraphPad Prism software (La Jolla, CA, USA). Data reported were from at least duplicate testing of antibody samples.

\section{Selection of escape mutants}

Isolation and selection of influenza virus escape mutants to mAbs was performed similar to described methods. ${ }^{26}$ Briefly, purified antibody $(\sim 2 \mathrm{mg})$ was incubated with $50 \mu \mathrm{l}$ of $\mathrm{A} /$ California/04/2009 $\left(\sim 10^{7} \mathrm{TCID}_{50} / \mathrm{ml}\right)$ virus in a total volume of $500 \mu \mathrm{l}$ for 1 hour at room temperature, diluted with an additional $500 \mu \mathrm{l}$ of PBS, and then $100 \mu \mathrm{l}$ of virus-antibody mixture was injected into each of 10 embryonated chicken eggs and incubated at $35^{\circ} \mathrm{C}$ for 2 days. Allantoic fluid from inoculated eggs was harvested individually and tested for the presence of virus by HA and for reduced $\mathrm{HI}$ activity of the original monoclonal antibody. Viruses that were inhibited $\sim 10$-fold less efficiently than A/California/04/2009 were cloned by limiting dilution in embryonated chicken eggs. Consensus nucleotide sequences of viral HAs were determined by direct 
DNA-sequencing of RT-PCR products and compared with those of the parental virus stock.

\section{Three-dimensional visualization of HA}

To visualize the mutations in the HA molecule of the virus escape mutants, the crystal structure of the HA of A/California/04/2009 was used (PDB ID: 3LZG; MMDB ID: $81425){ }^{27}$ Three-dimensional visualization was conducted using Cn3D-4.3 software. ${ }^{28}$

\section{Results}

\section{Generation and initial characterization of $\mathrm{mAbs}$ to A/California/4/2009 HA}

Mammalian-derived VLPs containing the HA of the pandemic influenza H1N1 A/California/04/2009 virus were used to immunize mice and generate a panel of hybridoma clones secreting mAbs to HA. Eleven antibodies bound to A/California/04/2009 HA in an ELISA using the H1 VLP that was the immunizing antigen for hybridoma production (Table 1) and were selected for further characterization. All of the mAbs but 2 (2E10 and 5F4) bound to virus HA when formaldehyde-inactivated A/California virus was used as the ELISA antigen (Table 1). Interestingly, one of these antibodies (2E10) also bound to VLPs containing the H5N1 A/Vietnam/1203/2004 HA (data not shown). None of the $11 \mathrm{mAbs}$ bound inactivated seasonal $\mathrm{H} 1 \mathrm{~N} 1$ virus (A/Solomon Islands/3/2006) or H3N2 (A/Uruguay/716 /2007) in this type of assay (data not shown). When each $\mathrm{mAb}$ was used in an ELISA format to capture virus, inactivated A/California/04/2009 virus bound strongly to all of the antibodies except 2E10 and 5F4. Under the conditions used, with each $\mathrm{mAb}$ used at a coating concentration of $1 \mu \mathrm{g} / \mathrm{ml}$ and inactivated virus at a starting concentration of $2 \mu \mathrm{g} / \mathrm{ml}$, there was weak binding to $2 \mathrm{E} 10$ and no apparent binding to $5 \mathrm{~F} 4$ (data not shown). Isotype analysis indicated that all $11 \mathrm{mAbs}$ were of the IgG2a subclass (data not shown).

Immunofluorescence microscopy was used to examine the ability of each monoclonal antibody to bind to virusinfected cells. MDCK cells were infected with A/California/04/2009, fixed at 3 days post-infection with $4 \%$ paraformaldehyde, and then stained with each monoclonal antibody (Table 1; Figure S1). Under these conditions, no fluorescence staining was detected for three antibodies (2E10, 5F4, and 1C5). Fluorescence staining was detected using the other eight antibodies, three of which (1A8, $5 \mathrm{C} 12$, and $4 \mathrm{~F} 8$ ) appeared somewhat more intense than the other five.

Each of the $11 \mathrm{~A} /$ California/04/2009 mAbs was assessed for binding of HA by Western blotting under reducing and non-reducing conditions at equivalent antibody

Table 1. Characterization of mAbs to $A / C a$ alifornia/04/2009 HA

\begin{tabular}{|c|c|c|c|c|c|c|}
\hline \multirow[b]{2}{*}{ Antibody } & \multicolumn{2}{|c|}{ Neutralization } & \multicolumn{2}{|l|}{ ELISA } & \multicolumn{2}{|c|}{ Western } \\
\hline & Titer* & HI titer** & H1 VLP*** & Inactivated virus & $\mathbf{R} / \mathrm{NR}^{\dagger}$ & $\mathbf{I F}^{+\dagger}$ \\
\hline $4 \mathrm{~F} 8$ & 691 & 1722 & $256 \mathrm{~K}$ & $512 \mathrm{~K}$ & $++/++$ & ++ \\
\hline $1 \mathrm{~A} 8$ & 453 & 1722 & $256 K$ & $256 \mathrm{~K}$ & $+/++$ & ++ \\
\hline $5 C 12$ & 453 & 1218 & $128 \mathrm{~K}$ & $256 K$ & $++/++$ & ++ \\
\hline $1 \mathrm{G} 10$ & 333 & 320 & $32 K$ & $128 \mathrm{~K}$ & $++/++$ & + \\
\hline 4A10 & 101 & 4 & $256 \mathrm{~K}$ & $128 \mathrm{~K}$ & $++/++$ & + \\
\hline 3A7 & 99 & 6 & $128 \mathrm{~K}$ & $128 \mathrm{~K}$ & $++/++$ & + \\
\hline $1 F 11$ & 67 & 9 & $256 K$ & $128 \mathrm{~K}$ & $++/++$ & + \\
\hline $3 \mathrm{G} 4$ & 32 & 19 & $128 \mathrm{~K}$ & $128 \mathrm{~K}$ & $++/++$ & + \\
\hline $1 C 5$ & 21 & 7 & $64 \mathrm{~K}$ & $32 \mathrm{~K}$ & $+/++$ & -- \\
\hline $2 \mathrm{E} 10$ & 5 & 11 & $256 \mathrm{~K}$ & $<1 \mathrm{~K}$ & $--/+$ & -- \\
\hline $5 F 4$ & 5 & 2 & $128 \mathrm{~K}$ & $<1 \mathrm{~K}$ & $--/--$ & -- \\
\hline$\beta$-gal & 5 & 2 & $<1 \mathrm{~K}$ & $1 \mathrm{~K}$ & NA & -- \\
\hline
\end{tabular}

VLP, virus-like particles; GMT, geometric mean titer; mAbs, monoclonal antibodies

${ }^{*} \mathrm{GMT}$ of the antibody neutralization of A/California/04/2009 infection of MDCK cells determined by microneutralization assay; initial mAb concentration $4 \mathrm{mg} / \mathrm{ml}$

${ }^{* *} \mathrm{GMT}$ of the antibody inhibition of A/California/04/2009 hemagglutination of chicken red blood cells; initial mAb concentration $0.2 \mathrm{mg} / \mathrm{ml}$.

$* * *$ Endpoint titer - highest dilution of antibody (initial concentration of $4 \mathrm{mg} / \mathrm{ml}$ ) giving an absorbance value $(405 \mathrm{~nm})>0.050$ and greater than the highest dilution of a matched dilution of control antibody of the same isotype; $K=1000$; antigens for capture (VLPs and inactivated whole influenza virus) used at $10 \mu \mathrm{g} / \mathrm{ml}$.

"Relative intensity of monoclonal antibody binding of influenza HA in Western blot analysis under reducing (R) and non-reducing (NR) conditions

${ }^{\dagger}$ Relative immunofluorescence intensity of monoclonal antibody binding to A/California/04/2009-infected MDCK cells. 
concentrations (Table 1; Figure 1). Seven of the mAbs bound to A/California HA well under reducing conditions, identifying an $\mathrm{HAl}$ of $\sim 55 \mathrm{kDa}$ (Figure 1A). Under the same conditions, two other mAbs (1A8 and 1C5) appeared to bind to HAl weakly; binding of HAl by the remaining two antibodies (2E10 and 5F4) was not detectable. Under non-reducing conditions (Figure 1B), all antibodies bound HA0 ( $\sim 70 \mathrm{kDa})$ except for 5F4. None of the 11 antibodies bound to the HA of pandemic H5N1 (A/Indonesia/5/05), seasonal H1N1 (A/Brisbane/59/2007), or H3N2 (A/Brisbane/10/2007) under either reducing or non-reducing conditions (Figure 1, lanes 1-3).

\section{Antigenic characterization of pandemic H1N1 mAbs by $\mathrm{VN}$ and $\mathrm{HI}$}

The panel of HA mAbs was evaluated for their ability to neutralize virus infectivity and inhibit virus hemagglutination of chicken red blood cells (Table 1). A microneutralization assay of A/California/04/2009 was performed using all mAbs at equivalent antibody concentration $(4 \mathrm{mg} / \mathrm{ml})$. Two antibodies (2E10 and 5F4) had no neutralizing antibody activity $(<1: 10)$ at the highest concentration of antibody. A range of neutralizing activities was measured for the other mAbs with four (4F8, 1A8, 5C12, and $1 \mathrm{G} 10$ ) exhibiting noticeably higher neutralization titers than the

A
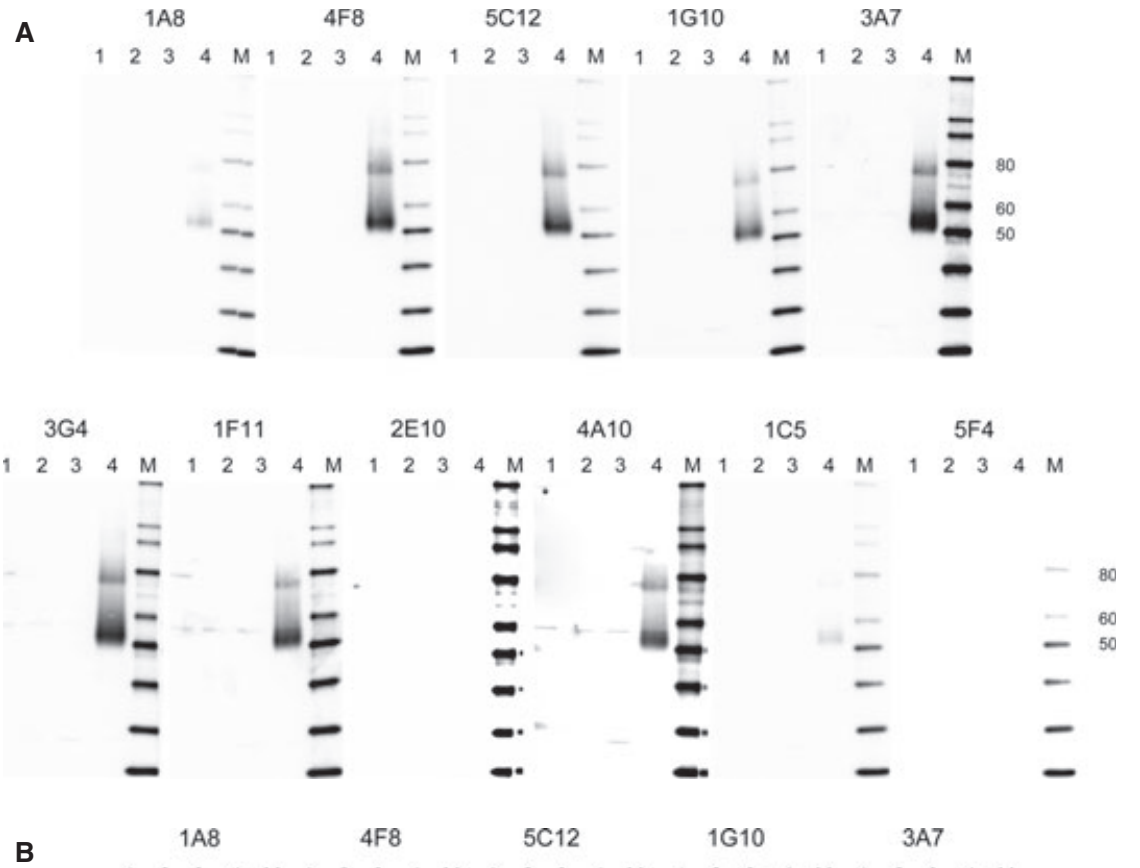
$\begin{array}{lllllllllllllllllllllllll}1 & 2 & 3 & 4 & M & 1 & 2 & 3 & 4 & M & 1 & 2 & 3 & 4 & M & 1 & 2 & 3 & 4 & M & 1 & 2 & 3 & 4 & M\end{array}$
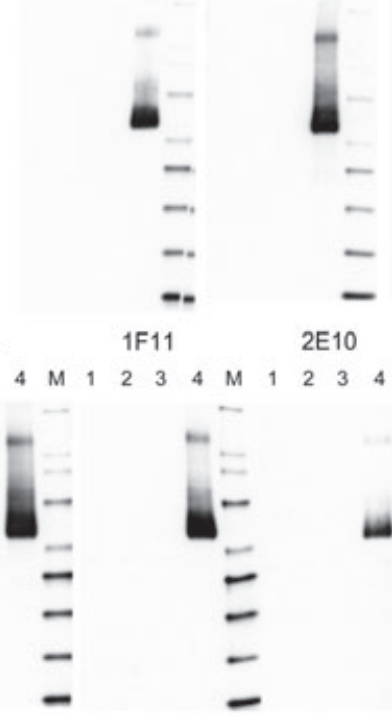
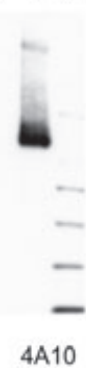

$4 \mathrm{~A} 10$
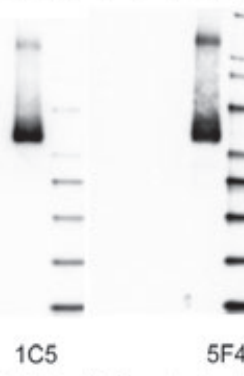

$5 \mathrm{~F} 4$

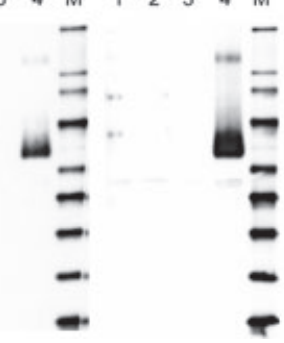

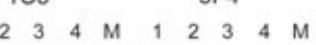

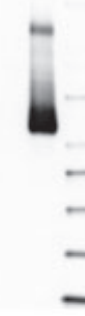

Figure 1. Monoclonal antibody detection of influenza HA in Western blot analysis. Viral proteins from inactivated influenza viruses were resolved by SDS-PAGE under reducing (A) and non-reducing conditions (B) and probed in Western blots with individual monoclonal antibodies $(2 \mu \mathrm{g} / \mathrm{ml})$. Lane 1 H5N1 (A/Indonesia/5/05); lane 2 - H1N1 (A/Brisbane/59/2007); lane $3-\mathrm{H} 3 \mathrm{~N} 2$ (A/Brisbane/10/2007); lane 4 - H1N1 (A/California/04/2009). M, molecular weight markers. 
others. When the panel of mAbs was analyzed in a HI assay using A/California/04/2009, the four antibodies with the highest neutralization titers $(4 \mathrm{~F} 8,1 \mathrm{~A} 8,5 \mathrm{C} 12$, and $1 \mathrm{G} 10)$ also presented high levels of $\mathrm{HI}$ activity. Low $\mathrm{HI}$ titers were measured for the remaining seven H1N1 HA mAbs.

Further characterization of the neutralizing potential of the panel of mAbs was carried out using a pseudotype neutralization assay (Table 2). Retroviral pseudotypes expressing various influenza $\mathrm{HA}$ proteins were incubated with individual antibodies, followed by infection of $293 \mathrm{~T}$ cells and measurement of vector expressed luciferase. In general, the results of the A/California pseudotype VN were consistent with the results of the microneutralization assay. The four antibodies with the highest pseudotype neutralization titers were the same four antibodies with the highest microneutralization and $\mathrm{HI}$ titers $(4 \mathrm{~F} 8,1 \mathrm{~A} 8,5 \mathrm{C} 12$, and 1G10). The pseudotype $\mathrm{VN}$ assay also facilitated analysis of the capacity of each monoclonal antibody to neutralize other influenza HA types. These studies demonstrated that none of the A/California HA antibodies could neutralize recent seasonal H1N1 HA-containing pseudotype viruses including A/New Caledonia/20/1999, A/Solomon Islands/3/2006, and A/Brisbane/59/2007 (data not shown). On the other hand, pseudotype viruses containing the influenza HA from A/South Carolina/1918 or A/New Jersey/1976 were neutralized to some degree by mAbs $4 \mathrm{~F} 8$, $1 \mathrm{~A} 8,5 \mathrm{C} 12$, and $1 \mathrm{G} 10$, and $4 \mathrm{~F} 8$ and $1 \mathrm{~A} 8$, respectively, indicating the closer relatedness of these viruses to the pandemic A/California/04/2009 pandemic virus (Table 2).

Table 2. Monoclonal antibody neutralization of H1N1 HAcontaining pseudoviruses

\begin{tabular}{|c|c|c|c|}
\hline \multirow[b]{2}{*}{ Antibody } & \multicolumn{3}{|c|}{ Neutralization titer $(\mu \mathrm{g} / \mathrm{ml})^{*}$} \\
\hline & A/California/2009 & $\mathrm{A} / \mathrm{SC} / 1918$ & A/NJ/1976 \\
\hline $4 \mathrm{~F} 8$ & 0.008 & $0 \cdot 106$ & $7 \cdot 299$ \\
\hline $1 \mathrm{~A} 8$ & 0.005 & $0 \cdot 114$ & $1 \cdot 703$ \\
\hline $5 C 12$ & 0.009 & $0 \cdot 157$ & $>40$ \\
\hline $1 \mathrm{G} 10$ & $0 \cdot 18$ & $0 \cdot 704$ & $>40$ \\
\hline $4 \mathrm{~A} 10$ & 1.98 & $>40$ & $>40$ \\
\hline $3 A 7$ & 0.54 & $28 \cdot 841$ & $>40$ \\
\hline 1F11 & 0.68 & $>40$ & $>40$ \\
\hline $3 \mathrm{G} 4$ & 0.66 & $21 \cdot 157$ & $>40$ \\
\hline $1 C 5$ & $4 \cdot 5$ & $33 \cdot 4$ & $>40$ \\
\hline $2 \mathrm{E} 10$ & $1 \cdot 25$ & $>40$ & $>40$ \\
\hline $5 F 4$ & $12 \cdot 32$ & N/A & N/A \\
\hline
\end{tabular}

*Antibody concentration resulting in a $95 \%$ reduction in relative luciferase units of retroviral pseudotypes expressing the indicated influenza virus $\mathrm{HA}$.

\section{Protection afforded by passive transfer of pandemic H1N1 mAbs}

To assess the protective capacity of the A/California/04/2009 HA mAbs, mice were passively transferred with antibodies prior to challenge with $\mathrm{A} / \mathrm{Mex}-$ ico/4108/2009, a relatively virulent strain of the 2009 pandemic H1N1 virus. Antibodies or PBS was injected intravenously into groups of mice 4 hours before intranasal challenge with $1 \mathrm{LD}_{50}$ of wild-type $\mathrm{A} / \mathrm{Mexico} / 4108 / 2009$. Mice were monitored for 2 weeks for morbidity (body weight loss) and mortality. Table 3 shows the results of two independent experiments evaluating the entire panel of H1N1 HA mAbs for their ability to provide passive protection against viral challenge. Transfer of PBS or an unrelated antibody ( $\beta$-Gal) failed to protect mice from death (40-60\% mortality) or morbidity. Average maximal weight loss in these groups approached $30 \%$ and the duration of $\geq 10 \%$ weight loss persisted for nearly 10 days over the course of the 2-week observation period. In contrast, the three antibodies with the highest neutralization and $\mathrm{HI}$ titers $(4 \mathrm{~F} 8,1 \mathrm{~A} 8$, and $5 \mathrm{C} 12)$ provided substantial protection against challenge. None of the mice in the groups that received these antibodies died, and the average maximal body weight loss never exceeded $10 \%$.

There was no obvious evidence of protection afforded by six of the mAbs (1G10, 4A10, 3A7, 1F11, 3G4, and 2E10) with several deaths in each group, average maximal weight loss of more than $20 \%$, and a duration of $\geq 10 \%$ weight loss of nearly 10 days. Interestingly, although neither of the mAbs $1 \mathrm{C} 5$ and $5 \mathrm{~F} 4$ had appreciable neutralizing or $\mathrm{HI}$ titers against $\mathrm{A} /$ California/04/2009 (Tables 1 and 2), transfer of these mAbs provided some protection against virus challenge, with no deaths observed in these groups. The average maximal body weight loss never exceeded 15\% in these mice, with only a few days in which average weight loss exceeded 10\%. Follow-up experiments using different preparations and concentrations of antibody confirmed their capacity to provide some protection against challenge (data not shown).

\section{Monoclonal antibody epitope mapping}

A competition ELISA experiment was designed to determine whether the four antibodies exhibiting the highest $\mathrm{HI}$ activity (4F8, 1A8, 5C12, and 1G10) competed for the same epitope(s). Purified 5C12 antibody was labeled with HRP and used as a detection antibody for bound VLPs containing the HA of A/California/04/2009. The amount of each unlabeled antibody required to inhibit labeled-5C12 binding by $50 \%$ was determined. The inhibitory concentration $50 \%\left(\mathrm{IC}_{50}\right)$ for the $\mathrm{HI}$ antibodies $4 \mathrm{~F} 8,1 \mathrm{~A} 8,5 \mathrm{C} 12$, and $1 \mathrm{G} 10$ was $0.03,0.31,0 \cdot 12$, and $0.63 \mu \mathrm{g} / \mathrm{ml}$, respectively. All of the remaining H1N1 HA mAbs had an $\mathrm{IC}_{50}>10 \mu \mathrm{g} / \mathrm{ml}$. 
Table 3. Protective effect of $A / C$ alifornia/04/2009 monoclonal antibodies

\begin{tabular}{|c|c|c|c|c|c|c|}
\hline \multirow[b]{2}{*}{ Antibody } & \multicolumn{2}{|c|}{ Survival } & \multicolumn{2}{|c|}{ Maximal body weight loss (\%)* } & \multicolumn{2}{|c|}{$\begin{array}{l}\text { Duration of } \geq 10 \% \text { body } \\
\text { weight loss (days)** }\end{array}$} \\
\hline & Exp 1 & $\operatorname{Exp} 2$ & Exp 1 & Exp 2 & Exp 1 & Exp 2 \\
\hline $4 \mathrm{~F} 8$ & $5 / 5$ & $5 / 5$ & $5 \cdot 9 \pm 2 \cdot 9$ & $7 \cdot 6 \pm 2 \cdot 8$ & 0 & 0 \\
\hline $1 \mathrm{~A} 8$ & $5 / 5$ & $5 / 5$ & $5 \cdot 9 \pm 2 \cdot 3$ & $9 \cdot 8 \pm 2 \cdot 4$ & 0 & 0 \\
\hline $5 C 12$ & $5 / 5$ & $5 / 5$ & $5 \cdot 5 \pm 1 \cdot 6$ & $3 \cdot 9 \pm 1 \cdot 2$ & 0 & 0 \\
\hline $1 \mathrm{G} 10$ & $3 / 5$ & $5 / 5$ & $31 \cdot 3 \pm 2 \cdot 6$ & $24 \cdot 2 \pm 6 \cdot 2$ & 10 & 10 \\
\hline $4 \mathrm{~A} 10$ & $4 / 5$ & $5 / 5$ & $26 \cdot 8 \pm 4 \cdot 8$ & $23 \cdot 6 \pm 5 \cdot 2$ & 10 & 8 \\
\hline $3 \mathrm{~A} 7$ & $4 / 5$ & $3 / 5$ & $28 \cdot 0 \pm 2 \cdot 9$ & $23 \cdot 0 \pm 4 \cdot 9$ & 10 & 8 \\
\hline $1 \mathrm{~F} 11$ & $2 / 5$ & $3 / 5$ & $33 \cdot 1 \pm 4 \cdot 5$ & $25 \cdot 3 \pm 3 \cdot 0$ & 10 & 10 \\
\hline $3 \mathrm{G} 4$ & $3 / 5$ & $2 / 5$ & $32 \cdot 9 \pm 1 \cdot 4$ & $33 \cdot 7 \pm 0 \cdot 7$ & 10 & 10 \\
\hline $1 C 5$ & $5 / 5$ & $5 / 5$ & $11 \cdot 7 \pm 1 \cdot 6$ & $14 \cdot 5 \pm 4 \cdot 7$ & 2 & 5 \\
\hline $2 \mathrm{E} 10$ & $1 / 5$ & $2 / 5$ & $33 \cdot 9 \pm 4 \cdot 0$ & $29 \cdot 1 \pm 4 \cdot 3$ & 10 & 10 \\
\hline $5 F 4$ & $5 / 5$ & $5 / 5$ & $10 \cdot 8 \pm 4 \cdot 2$ & $6 \cdot 3 \pm 2 \cdot 9$ & 3 & 0 \\
\hline PBS & $2 / 5$ & $2 / 5$ & $25 \cdot 7 \pm 6 \cdot 9$ & $29 \cdot 6 \pm 2 \cdot 1$ & 6 & 10 \\
\hline$\beta$-gal & ND & $3 / 5$ & ND & $29 \cdot 5 \pm 1 \cdot 7$ & ND & 10 \\
\hline
\end{tabular}

The results suggested that the four mAbs with the strongest HI activity were directed toward the same or a nearby epitope.

Escape mutants to A/California/04/2009 were selected by incubation of the virus with mAbs $4 \mathrm{~F} 8$ and $5 \mathrm{C} 12$. Viruses that were inhibited in the $\mathrm{HI}$ assay $\sim 10$-fold less efficiently than A/California/04/2009 were cloned by limiting dilution. Eleven independently isolated escape mutants were obtained, sequenced, and compared with the sequence of the parent virus. Two separate mutations were identified, either a D127E or a G155E (H1 numbering used throughout the text), both located near the antigenic site Sa. Figure 2A shows the sequence of the two escape mutants. Both mutations are located close to each other near the head of the HA molecule as shown in the 3-dimensional HA structure diagram (Figure 2B). Compared with A/California/04/2009, each mutant virus was more resistant to neutralization by mAbs 4F8, 5C12, and 1G10. Similarly, HI titers for these antibodies were greatly reduced for the escape mutants compared with A/California/04/2009 (Table 4). In contrast, the neutralizing activity of $4 \mathrm{~A} 10$, which apparently does not share this epitope based on the ELISA competition assay, was not affected by either mutation. Interestingly, the same amino acid changes identified in the escape mutants were present in A/South Carolina/1/1918 (D127E - Genbank accession no. AAD17229) and A/New Jersey/1976 (D127E and G155E - Genbank accession no. ABQ44394) HAs and thus may explain the poor neutralization of pseudotype viruses bearing these HAs by the A/California/04/2009 mAbs (Table 2). Finally, we tested whether antibodies generated by vaccination with pandemic $2009 \mathrm{H} 1 \mathrm{~N} 1$ vaccines still recognized the D127E and G155E escape mutants. Pre- and post-vaccination sera were obtained from eight adults receiving seasonal vaccine containing the recommended A/California/07/2009-like virus strain and tested in an $\mathrm{HI}$ assay using A/California/04/2009 and the two A/California escape mutants with either the D127E or G155E mutation. All pre-vaccination sera had $\mathrm{HI}$ titers below the level of detection (1:20) to A/California/04/2009 and both A/California escape mutants. Seven of the eight post-vaccination sera had HI titers >1:160 to A/California/04/2009; one post-vaccination HI titer was below the level of detection. However, there was no difference in post-vaccination $\mathrm{HI}$ titers between A/California/04/2009 and either escape mutant for any of the tested sera (data not shown), suggesting that the single mutations were not sufficient to abrogate a protective antibody response to the vaccine.

\section{Discussion}

This study describes the initial characterization of a panel of murine mAbs to the HA of A/California/04/2009. The antibodies exhibit a range of properties and thus have a variety of uses that will facilitate characterization of the $\mathrm{A}(\mathrm{H} 1 \mathrm{~N} 1) \mathrm{pdm} 09$ virus HA. The panel of hybridoma clones 
Figure 2. Location of $\mathrm{HA}$ amino acid changes in A/California/04/2009 escape mutants. (A) Sequence alignment of A/California/04/2009 and escape mutants selected by monoclonal antibodies to HA. The antigenic region $\mathrm{Sa}$ is shown in color. The amino acid changes identified in the escape mutants are shaded. Amino acid sequences in the same region of $A /$ South Carolina/1/1918 and $A /$ New Jersey/1976 are aligned for comparison. Amino acid numbering is as previously described. ${ }^{27}$ (B) Antigenic structure of $A / C a l i f o r n i a ~ H A$. The locations of the two amino acid changes identified in the escape mutants are highlighted.
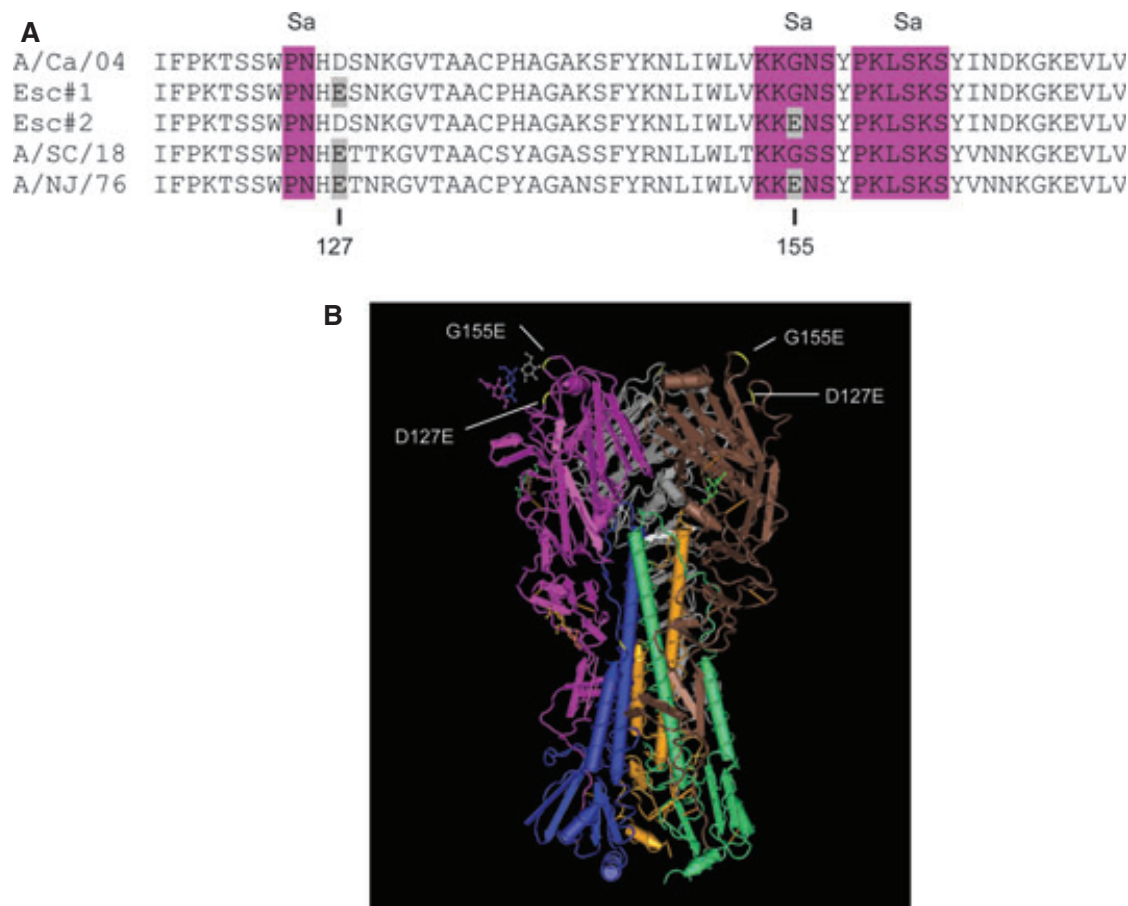

Table 4. Neutralization and $\mathrm{HI}$ titers of mAbs to A/California/04/2009 escape mutants

\begin{tabular}{|c|c|c|c|c|c|c|c|c|c|c|}
\hline \multirow[b]{2}{*}{ Virus } & \multicolumn{5}{|c|}{ Neutralization titer* } & \multicolumn{5}{|c|}{ HI titer** } \\
\hline & 4F8 & $5 C 12$ & $1 \mathrm{G} 10$ & 4A10 & $5 \mathrm{~F} 4$ & 4F8 & $5 \mathrm{C} 12$ & $1 \mathrm{G} 10$ & $4 A 10$ & $5 \mathrm{~F} 4$ \\
\hline $\mathrm{A} / \mathrm{CA} / 04 / 2009$ & 640 & 381 & 453 & 48 & $<10$ & 912 & 1024 & 256 & 4 & $<2$ \\
\hline Mutant D127E & 48 & 34 & 67 & 113 & $<10$ & 8 & 9 & 7 & 6 & $<2$ \\
\hline Mutant G155E & $<40$ & $<40$ & $<40$ & 57 & $<10$ & $<2$ & $<2$ & $<2$ & 4 & $<2$ \\
\hline
\end{tabular}

$\mathrm{HI}$, hemagglutination inhibition; GMT, geometric mean titer; mAbs, monoclonal antibodies.

${ }^{*} \mathrm{GMT}$ of the antibody neutralization titer of A/California/04/2009 or escape mutants D127E and G155E as determined by microneutralization assay. Limit of detection for neutralization was 40 for 4F8, 5C12, and 1G10; 10 for $4 \mathrm{~A} 10$ and 5F4.

**GMT of the antibody HI titer of A/California/04/2009 or escape mutants D127E and G155E. Limit of detection for HI was 2.

was generated using mammalian-derived VLP containing the HA of the A/California/04/2009 virus as the immunogen. These VLP particles were produced from a recombinant MVA vector expressing only the HA of A/California/04/2009. Such VLPs have been shown to have properties in common with live influenza virus including their ability to elicit neutralizing antibodies and provide protection against influenza challenge in animals. ${ }^{18}$ By using this approach for immunization, the expressed virus $\mathrm{HA}$ is presented in its native form as in a virus particle. Also, because no other influenza virus proteins are present during immunization, the initial screening and counterscreening to select the desired hybridomas are simplified by using whole influenza virus, including unrelated influenza viruses, to identify antibodies recognizing the specific HA. An immunization approach using VLPs would have an added advantage for highly pathogenic influenza viruses because there would be no virus containment issues to address during the animal procedures.

Initial characterization using ELISA, immunofluorescence, and Western blot analysis identified mAbs useful for several practical applications. By all methodologies, the panel of mAbs appeared highly specific for the HA of A/California/04/2009 with no cross-reactivity to the HA of seasonal H1N1 viruses. ELISA results using a variety of influenza viruses or VLPs as capture antigen demonstrated 
sensitivity and specificity of antibody binding; no crossreactivity of the mAbs with other seasonal H1N1 or H3N2 viruses was detected. Only one $\mathrm{mAb}, 2 \mathrm{E} 10$, showed some cross-reactivity with a VLP containing the HA of an H5N1 virus (data not shown). Eight of the $11 \mathrm{mAbs}$ were suitable for use in immunofluorescence studies and three of these $(1 \mathrm{~A} 8,5 \mathrm{C} 12$, and $4 \mathrm{~F} 8)$ appeared particularly sensitive. In Western blot analysis under non-reducing conditions, all of the mAbs except for 5F4 bound to A/California/04/2009 $\mathrm{HA}$, whereas none of the antibodies bound to HA from seasonal or pandemic influenza strains. It was possible to conclude that nine of the $11 \mathrm{mAbs}$ recognized epitopes on the HAl portion of the HA because they recognized HAl but not HA2 under Western blot reducing conditions. Interestingly, binding of some mAbs (e.g., 1C5) was rather weak in reducing conditions compared with the binding under non-reducing conditions, suggesting that such antibodies might be directed to epitopes that are conformationally sensitive.

Hemagglutination inhibition and $\mathrm{VN}$ by antibodies to influenza HA are considered important functional properties that contribute to protection. We used mAbs with HI and neutralizing activity to identify and characterize potentially important antigenic epitopes of the A/California/04/2009 HA. There were four mAbs with significant measurable HI activity, and these four antibodies were the strongest neutralizing antibodies in both standard virus microneutralization assays and pseudotype neutralization assays. In general, both types of neutralization assays appeared more sensitive than HI. Whereas only four mAbs had appreciable HI activity even when the highest practical concentrations of antibody were tested, several of the other mAbs had lower but easily quantifiable neutralization activity in both assays. The absolute endpoint titers determined for the mAbs in the pseudotype neutralization assay were much higher than the corresponding titers obtained in the microneutralization assay. Similar observations have been previously reported, and the differences are likely due in part to the different methodologies of the assays, particularly in the sensitivity of virus or pseudotype virus detection. However, it has also been suggested that the greater apparent sensitivity of the pseudotype neutralization assay may be the result of a lower density of HA molecules on the surface compared with the actual virus, making the HA more accessible to antibody when expressed on the pseudotype virus. ${ }^{24}$ Nevertheless, there was overall good agreement between the relative results of the two neutralization assays, particularly at the highest and lowest ranges of activity, although one $\mathrm{mAb}, 4 \mathrm{~A} 10$, appeared to have a higher neutralization activity in the microneutralization assay relative to the other mAbs than it did in the pseudotype assay.

Although many $\mathrm{A}(\mathrm{H} 1 \mathrm{~N} 1) \mathrm{pdm} 09$ viruses were not especially pathogenic in animal models of infection, some viruses such as $\mathrm{A} / \mathrm{Mexico} / 4108 / 2009$ are relatively virulent strains and can be used to assess protection afforded by vaccines and antibodies. In our mouse challenge experiments with this virus, we observed a $40 \%$ mortality and substantial morbidity as evidenced by maximal body weight loss of $\sim 30 \%$ and almost 10 days of weight loss $>10 \%$ of animal body weight. The three mAbs with the highest HI and neutralizing titers provided passive protection against virus challenge by all criteria (e.g., no deaths and no body weight loss $>10 \%$ ), suggesting a correlation between the functional properties of the mAbs measured in vitro (HI and neutralizing activity) and protection. Although $\mathrm{mAb}$ $1 \mathrm{G} 10$ had both $\mathrm{HI}$ and neutralizing activity, it appeared weaker in all three in vitro assays than the other three HI antibodies, which may explain its inability to provide protection against virus challenge in the passive transfer experiments. Interestingly, two of the mAbs with no appreciable $\mathrm{HI}$ activity or neutralizing activity in these in vitro assays showed evidence of protection in the passive transfer experiments. Although not as protective as the three $\mathrm{HI}$ mAbs (4F8, 1A8, and $5 \mathrm{C} 12$ ), both $1 \mathrm{C} 5$ and $5 \mathrm{~F} 4$ prevented death and lessened morbidity associated with challenge compared with PBS, control antibodies, and the other six mAbs. Multiple challenge experiments using different preparations and concentrations of antibody confirmed these observations. At this time, it is not clear how these two mAbs exert their protective effect. Antiviral mechanisms of antibody activity other than those measured in traditional $\mathrm{HI}$ and neutralization assays have been described, including complement-dependent lysis of infected cells ${ }^{29}$ and complement-mediated neutralization and enhancement of neutralization by serum components other than complement. ${ }^{30-33}$ We have conducted some preliminary complement-mediated neutralization experiments, and both 1C5 and 5F4 appear to have enhanced neutralizing activity in the presence of complement. However, their enhanced neutralization activities were no greater than those of the six mAbs that did not afford any protection in the passive transfer experiments (data not shown). Additional studies are planned to try to understand how $1 \mathrm{C} 5$ and $5 \mathrm{~F} 4 \mathrm{mAbs}$ interact with the virus and impart a protective effect in vivo.

Five major antigenic sites ( $\mathrm{Sa}, \mathrm{Sb}, \mathrm{Ca} 1, \mathrm{Ca} 2$, and $\mathrm{Cb}$ ) have been described in the HA of influenza H1N1. ${ }^{34,35}$ To identify the HA epitopes recognized by the neutralizing mAbs in our panel, we selected virus escape mutants by incubating A/California/04/2009 in the presence of individual mAbs. Cloned viruses that were resistant to $\mathrm{HI}$ by antibody were sequenced to identify mutations. Although 11 independently derived virus mutants were obtained, there were only two unique mutations, both near the antigenic site Sa. All of the strong $\mathrm{HI}$ and neutralizing mAbs had reduced titers for both escape mutants in HI and 
neutralization assays, as might be predicted from the ELISA competition studies, which suggested that the four antibodies $4 \mathrm{~F} 8,5 \mathrm{C} 12,1 \mathrm{~A} 8$, and $1 \mathrm{G} 10$ recognized the same or a nearby epitope on the HA. There was some indication, however, that the recognition site for these antibodies might not be identical. During the selection of escape mutants to $5 \mathrm{C} 12$, four of five independently isolated mutations had the D127E mutation and one isolate had the G155E mutation. In contrast, during selection of escape mutants to $4 \mathrm{~F} 8$, all six independently isolated mutants were G155E. Interestingly, some human mAbs directed to the Sa region of the 1918 pandemic H1N1 virus HA have been shown to cross-react with the $\mathrm{A}(\mathrm{H} 1 \mathrm{~N} 1) \mathrm{pdm} 09 \mathrm{HA}^{36}$ Although the epitopes recognized by the human mAbs appear to be different from the epitopes identified in the present study, the results strongly suggest that the Sa region of both H1N1 viral HAs is an important target for humoral immunity.

Since the emergence of the $\mathrm{A}(\mathrm{H} 1 \mathrm{~N} 1) \mathrm{pdm} 09$ virus, very few genetic or antigenic changes in the virus HA have been documented. Consequently, the recommended virus strain for inclusion in the seasonal influenza vaccine has remained an $\mathrm{A} /$ California/07/2009-like virus for the 3 years since the pandemic, including the next 2012-2013 northern hemisphere winter influenza season. ${ }^{37}$ Nevertheless, there have been some reports of virus isolates containing amino acid changes in the HA that have the potential to alter the antigenic properties of the virus. For example, only two amino acid changes in the Sa region (N125D and N156K) of virus isolates from Finland were sufficient to reduce the interaction of antibodies in convalescent sera or sera from vaccinated individuals as measured by $\mathrm{HI}^{38}$ There have also been a couple of reports describing adaptation and increased virulence of A/California/04/2009 in mice, ${ }^{39,40}$ and interestingly, both of the mutations identified in our mAb escape mutants (D127E and G155E) were also identified in these studies. In one study, a mouse adapted A/California/04/2009 virus contained amino acid mutations in several virus genes, including four in the HA, and exhibited increased virulence in mice. Although the mutations were not analyzed individually for their contribution to increased virulence, one of the four HA amino acid mutations was a G155E. ${ }^{39}$ In another study, mutations in two amino acids in the A/California/04/2009 HA, D131E (D127E in our numbering), and S186P had a significant impact on virulence in mice. ${ }^{40}$ By itself, the D131E (D127E) mutation appeared to result in a greater loss of animal body weight ( $11 \%$ versus $4 \%$ for $\mathrm{A} /$ Califor$\mathrm{nia} / 04 / 2009)$ and increased virus titers in the lungs of mice $(\sim 0.5 \mathrm{log})$. Ferret sera to A/California/04/2009 had reduced $\mathrm{HI}$ titer to a virus containing the D131E (D127E) mutation. In contrast, in our limited study with human vaccine sera, there were no differences in $\mathrm{HI}$ titers between
A/California/04/2009 and our D127E escape mutant, likely reflecting differences between the two methodologies using post-infection ferret sera and post-vaccination human sera.

In summary, this study characterizes a panel of murine mAbs to the HA of the $\mathrm{A}(\mathrm{H} 1 \mathrm{~N} 1) \mathrm{pdm} 09$ virus $\mathrm{A} /$ California/04/2009. Several antibodies with neutralizing and hemagglutination activity were identified, some of which were able to provide protection when administered passively before influenza challenge. The HA epitopes recognized by strongly neutralizing $\mathrm{mAbs}$ were identified by isolation of virus escape mutants and mapped near the antigenic site Sa. This panel of mAbs allowed comparison of antigenic and neutralizing epitopes of the HA of A/California/04/2009 to other H1N1 viruses, including recent seasonal isolates as well as A/South Carolina/1918 and A/New Jersey/1976 and may be useful in identifying important HA epitopes involved in future antigenic drift.

\section{Acknowledgements}

MDCK cells were a gift from Dr. Jacqueline Katz (CDC, Atlanta, GA, USA). We thank Dr. Maryna Eichelberger and Dr. Keith Peden for critical review of the manuscript. This work was supported in part by the Biomedical Advanced Research and Development Authority, Department of Health and Human Services. Rachel Friedman and Joseph Besho were participants of the Oak Ridge Institute for Science and Education program at CBER.

\section{References}

1 Dawood FS, Jain S, Finelli L et al. Emergence of a novel swine-origin influenza A (H1N1) virus in humans. N Engl J Med 2009; 360:2605-2615.

2 Shinde V, Bridges CB, Uyeki TM et al. Triple-reassortant swine influenza $A(H 1)$ in humans in the United States, 2005-2009. N Engl J Med 2009; 360:2616-2625.

3 Garten RJ, Davis CT, Russell CA et al. Antigenic and genetic characteristics of swine-origin 2009 A(H1N1) influenza viruses circulating in humans. Science 2009; 325:197-201.

4 Smith GJ, Vijaykrishna D, Bahl J et al. Origins and evolutionary genomics of the 2009 swine-origin H1N1 influenza A epidemic. Nature 2009; 459:1122-1125.

5 Vincent $\mathrm{AL}$, Lager $\mathrm{KM}, \mathrm{Ma} \mathrm{W}$ et al. Evaluation of hemagglutinin subtype 1 swine influenza viruses from the United States. Vet Microbiol 2006; 4:212-222.

6 Hancock K, Veguilla V, Lu X et al. Cross-reactive antibody responses to the 2009 pandemic H1N1 influenza virus. N Engl J Med 2009; 361:1945-1952.

7 LaBoissiere S, O'Hare P. Analysis of HCF, the cellular cofactor of VP16, in herpes simplex virus-infected cells. J Virol 2000; 74:99-109.

8 McCullers JA, Van De Velde LA, Allison KJ, Branum KC, Webby RJ, Flynn PM. Recipients of vaccine against the 1976 "swine flu" have enhanced neutralization responses to the 2009 novel H1N1 influenza virus. Clin Infect Dis 2010; 50:1487-1492.

9 Xie H, Li X, Gao J et al. Revisiting the 1976 "swine flu" vaccine clinical trials: cross-reactive hemagglutinin and neuraminidase antibodies 
and their role in protection against the $2009 \mathrm{H} 1 \mathrm{~N} 1$ pandemic virus in mice. Clin Infect Dis 2011; 53:1179-1187.

10 Wang $W$, Anderson CM, De Feo CJ et al. Cross-neutralizing antibodies to pandemic $2009 \mathrm{H} 1 \mathrm{~N} 1$ and recent seasonal H1N1 influenza A strains influenced by a mutation in hemagglutinin subunit 2. PLoS Pathog 2011;7:e1002081.

11 Ellebedy AH, Ducatez MF, Duan $S$ et al. Impact of prior seasonal influenza vaccination and infection on pandemic A (H1N1) influenza virus replication in ferrets. Vaccine 2011; 29:3335-3339.

12 Del Giudice G, Stittelaar KJ, van Amerongen G et al. Seasonal influenza vaccine provides priming for $\mathrm{A} / \mathrm{H} 1 \mathrm{~N} 1$ immunization. Sci Transl Med 2009; 1:12re1.

13 Manicassamy B, Medina RA, Hai R et al. Protection of mice against lethal challenge with 2009 H1N1 influenza A virus by 1918-like and classical swine H1N1 based vaccines. PLoS Pathog 2010; 6:e1000745.

14 Labrosse B, Tourdjman M, Porcher R et al. Detection of extensive cross-neutralization between pandemic and seasonal A/H1N1 Influenza Viruses using a pseudotype neutralization assay. PLOS ONE 2010; 5:e11036.

15 Plennevaux E, Sheldon E, Blatter M, Reeves-Hoche MK, Denis M. Immune response after a single vaccination against 2009 influenza A H1N1 in USA: a preliminary report of two randomised controlled phase 2 trials. Lancet 2010; 375:41-48.

16 Lee VJ, Tay JK, Chen Ml et al. Inactivated trivalent seasonal influenza vaccine induces limited cross-reactive neutralizing antibody responses against 2009 pandemic and 1934 PR8 H1N1 strains. Vaccine 2010; 28:6852-6857.

17 Xie $\mathrm{H}$, Jing $\mathrm{X}$, Li $\mathrm{X}$ et al. Immunogenicity and cross-reactivity of 2009-2010 inactivated seasonal influenza vaccine in US adults and elderly. PLOS ONE 2011; 6:e16650.

18 Schmeisser F, Adamo JE, Blumberg B et al. Production and characterization of mammalian virus-like particles from modified vaccinia virus Ankara vectors expressing influenza $\mathrm{H} 5 \mathrm{~N} 1$ hemagglutinin and neuraminidase. Vaccine 2012; 30:3413-3422.

19 Schmeisser F, Vodeiko GM, Lugovtsev VY, Stout RR, Weir JP. An alternative method for preparation of pandemic influenza strainspecific antibody for vaccine potency determination. Vaccine 2010; 28:2442-2449.

20 Earl P, Cooper N, Wyatt L, Moss B, Carroll M. Preparation of cell cultures and vaccinia virus stocks; in Ausubel FM, Brent $R$, Kingston RE, Moore DD, Seidman JG, Smith J et al. (eds): Current Protocols in Molecular Biology. New York: John Wiley and Sons, Inc., 1998; 16.16.1-16.16.13.

21 Palmer DF, Coleman MT, Dowdle WR, Schild GC. Advanced Laboratory Techniques for Influenza Diagnosis. Washington, DC: U.S. Department of Health, Education and Welfare, 1975.

22 Rowe T, Abernathy RA, Hu-Primmer J et al. Detection of antibody to avian influenza A (H5N1) virus in human serum by using a combination of serologic assays. J Clin Microbiol 1999; 37:937-943.

23 Wang W, Castelan-Vega JA, Jimenez-Alberto A, Vassell $R$, Ye $Z$, Weiss CD. A mutation in the receptor binding site enhances infectivity of 2009 H1N1 influenza hemagglutinin pseudotypes without changing antigenicity. Virology 2010; 407:374-380.

24 Wang W, Butler EN, Veguilla $V$ et al. Establishment of retroviral pseudotypes with influenza hemagglutinins from $\mathrm{H} 1, \mathrm{H} 3$, and $\mathrm{H} 5$ subtypes for sensitive and specific detection of neutralizing antibodies. J Virol Methods 2008; 153:111-119.

25 Wang W, Xie H, Ye Z, Vassell R, Weiss CD. Characterization of lentiviral pseudotypes with influenza H5N1 hemagglutinin and their performance in neutralization assays. J Virol Methods 2010; 165:305-310.

26 Gerhard W, Webster RG. Antigenic drift in influenza A viruses. I. Selection and characterization of antigenic variants of $A / P R / 8 / 34$ (HON1) influenza virus with monoclonal antibodies. J Exp Med 1978; 148:383-392.

27 Xu R, Ekiert DC, Krause JC, Hai R, Crowe JE Jr, Wilson IA. Structural basis of preexisting immunity to the $2009 \mathrm{H} 1 \mathrm{~N} 1$ pandemic influenza virus. Science 2010; 328:357-360.

28 Wang Y, Geer LY, Chappey C, Kans JA, Bryant SH. Cn3D: sequence and structure views for Entrez. Trends Biochem Sci 2000; 25:300302.

29 Terajima M, Cruz J, Co MD et al. Complement-dependent lysis of influenza a virus-infected cells by broadly cross-reactive human monoclonal antibodies. J Virol 2012; 85:13463-13467.

30 Beebe DP, Schreiber RD, Cooper NR. Neutralization of influenza virus by normal human sera: mechanisms involving antibody and complement. J Immunol 1983; 130:1317-1322.

31 Reno PW, Hoffmann EM. Enhancement of hemagglutination inhibition by complement. Infect Immun 1972; 6:945-951.

32 Feng JQ, Mozdzanowska K, Gerhard W. Complement component C1q enhances the biological activity of influenza virus hemagglutinin-specific antibodies depending on their fine antigen specificity and heavy-chain isotype. J Virol 2002; 76:1369-1378.

33 Mozdzanowska K, Feng J, Eid M, Zharikova D, Gerhard W. Enhancement of neutralizing activity of influenza virus-specific antibodies by serum components. Virology 2006; 352:418-426.

34 Caton AJ, Brownlee GG, Yewdell JW, Gerhard W. The antigenic structure of the influenza virus $A / P R / 8 / 34$ hemagglutinin ( $\mathrm{H} 1$ subtype). Cell 1982; 31(2 Pt 1):417-427.

35 Gerhard W, Yewdell J, Frankel ME, Webster R. Antigenic structure of influenza virus haemagglutinin defined by hybridoma antibodies. Nature 1981; 290:713-717

36 Krause JC, Tumpey TM, Huffman CJ et al. Naturally occurring human monoclonal antibodies neutralize both 1918 and 2009 pandemic influenza A (H1N1) viruses. J Virol 2010; 84:3127-3130.

37 World Health Organization. Recommended composition of influenza virus vaccines for use in the 2012-2013 northern hemisphere influenza season. Available at http://www.who.int/influenza/vaccines/ virus/recommendations/201202_recommendation.pdf (Accessed 13 Junel 2012).

38 Strengell $\mathrm{M}$, Ikonen $\mathrm{N}$, Ziegler $\mathrm{T}$, Julkunen I. Minor changes in the hemagglutinin of influenza $\mathrm{A}(\mathrm{H} 1 \mathrm{~N} 1) 2009$ virus alter its antigenic properties. PLOS ONE 2011; 6:e25848.

39 Ilyushina NA, Khalenkov AM, Seiler JP et al. Adaptation of pandemic H1N1 influenza viruses in mice. J Virol 2010; 84:8607-8616.

40 Ye J, Sorrell EM, Cai $Y$ et al. Variations in the hemagglutinin of the 2009 H1N1 pandemic virus: potential for strains with altered virulence phenotype? PLoS Pathog 2010; 6:e1001145.

\section{Supporting Information}

Additional Supporting Information may be found in the online version of this article:

Figure S1. Immunofluorescence of A/California/04/2009infected MDCK cells. 